\title{
Transatlantica
}

Revue d'études américaines. American Studies Journal

$2 \mid 2011$

Sport et société / Animals and the American

Imagination

\section{Photo League, Weegee et Cindy Sherman à New York}

Jewish Museum (4 novembre 2011 - 25 mars 2012) ; ICP (20 janvier - 2

septembre 2012) ; MoMa (26 février - 11 juin 2012)

Jean Kempf

\section{OpenEdition}

\section{Journals}

Édition électronique

URL : https://journals.openedition.org/transatlantica/5596

DOI : 10.4000/transatlantica.5596

ISSN : 1765-2766

Éditeur

Association française d'Etudes Américaines (AFEA)

Référence électronique

Jean Kempf, « Photo League, Weegee et Cindy Sherman à New York », Transatlantica [En ligne], 2 |

2011, mis en ligne le 11 juin 2012, consulté le 01 février 2023. URL : http://journals.openedition.org/ transatlantica/5596 ; DOI : https://doi.org/10.4000/transatlantica.5596

Ce document a été généré automatiquement le 1 février 2023.

Creative Commons - Attribution - Pas d'Utilisation Commerciale - Pas de Modification 4.0 International - CC BY-NC-ND 4.0

https://creativecommons.org/licenses/by-nc-nd/4.0/ 


\section{Photo League, Weegee et Cindy Sherman à New York}

Jewish Museum (4 novembre 2011 - 25 mars 2012) ; ICP (20 janvier - 2

septembre 2012) ; MoMa (26 février - 11 juin 2012)

Jean Kempf

\section{NOTE DE L'AUTEUR}

The Radical Camera : New York's Photo League, 1936-1951, du 4 novembre 2011 au 25 mars 2012 au Jewish Museum de New York

Weegee : Murder Is My Business, du 20 janvier au 2 septembre 2012 à l'International Center for Photography, New York

Cindy Sherman, du 26 février au 11 juin 2012 au Museum of Modern Art, New York

1 Trois expositions photographiques sont actuellement visibles à New York, qu'il peut être intéressant de considérer côte à côte. La Photo League au Jewish Museum, Murder is my Business de Weegee à l'International Center of Photography et Cindy Sherman au MoMA. Les trois sont des expositions ambitieuses dans leur propos, quoique de taille et de facture fort différentes.

2 Très belle exposition à tous points de vue, la Photo League au Jewish Museum est présentée dans un espace agréable et parfaitement calibré, suffisamment spacieux sans être trop pesant, offrant une sélection intelligente, innovante et instructive des images réalisées entre 1936 et 1951 par cette célèbre association qui marqua le reportage en tant que genre, et, au-delà, tout l'art photographique des années 30 aux années 60 . À côté de tirages d'époque et contemporains présentés de façon claire, les commissaires ont placé divers documents (revue de la League, photo des réunions et des photographes eux-mêmes), ainsi que des textes didactiques qui expliquent au visiteur non spécialiste le fonctionnement de cette association coopérative de production et d'éducation photographiques. Certes, l'attaque dont elle est la cible pendant le maccarthysme, et qui a conduit à sa disparition, est très présente dans cet historique 
mais au-delà, l'exposition met en évidence ce qui fit la spécificité de sa production et sa qualité : un fort ancrage urbain, une culture d'immigration récente (souvent mais pas exclusivement juive), une immense foi en ce que l'Amérique pouvait alors offrir, ainsi qu'un enthousiasme qui dépasse le simple fait de l'âge des participants. Dans ce bouillon de culture du New York des années 30, on voit s'inventer une photographie américaine qui veut à la fois tenir de l'exigence documentaire et esthétique («le politique et la poétique " écrit Mason Klein dans le catalogue), mélange détonant et beau de Neue Sachlich et d'Ash Can school, de constructivisme et de précisionnisme, de presse et d'art. Bref, on découvre ce chaînon manquant de la photographie, entre l'esthétique froide d'un Evans ou d'un Sheeler d'un côté et l'efficacité de la FSA de l'autre, ce qui fera la force de l'expression photographique américaine des années 50 avec un Eugene Smith (membre de la Photo League) et plus tard les Robert Frank, Friedlander et alii. Dans cette exposition, le plaisir de la découverte d'images peu connues ne cède en rien à celui de la compréhension d'une continuité qui éclaire bien des aspects de l'histoire de la photographie américaine.

3 L'exposition Weegee à l'ICP est, elle, centrée sur Naked City, l'ouvrage autobiographique du célèbre reporter, lui-même issu de la Photo League, mais reprend dans son titre celui d'un autre de ses ouvrages, Murder is my business, qui résume le positionnement de celui qui fut autant qu'un photographe un habile vendeur et une star du reportage, de même qu'une sorte de caméléon s'identifiant à la pègre qu'il côtoyait. Le personnage est d'ailleurs très présent avec des artefacts lui ayant appartenu, et même la reconstitution de sa chambre/bureau et des images de son célèbre coffre/atelier de voiture. On n'apprend guère de nouveau sur le "cas Weegee ", car si l'exposition est fort bien montée, elle participe à l'hagiographie classique qu'il a lui-même fabriquée toute sa vie. Rien ne manque à la légende. Avec lui, c'est la star médiatique, l' « urban cowboy ", mélange de Mickey Spillane et de Raymond Chandler, qui émerge dans le champ du photojournalisme. Avec Weegee, le photographe cesse d'être un anonyme sans pour autant se revendiquer comme auteur. Ici, on voit un homme en apparence mû par le scoop le plus cru (scènes de meurtre ou d'accident qui ne cache rien des détails) qu'il vend aux grands tabloïds de l'époque, qui, en même temps, ne cesse de jouer la comédie, d'incarner (pour des besoins en apparence didactiques) le rôle du malfrat, passant entre les mains de la police, se mettant en scène dans un chemin de croix contemporain où il est tour à tour arrêté, fiché, interrogé et incarcéré. Mégalomane mais surtout éperdument narcissique, cet immigrant qui incarne l'un des aspects les plus folkloriques mais aussi sombres de la grande ville américaine surprend moins qu'il ne confirme le glissement vers la figure du photographe et l'escalade du scoop. Le voyeurisme de son objectif, et surtout de son flash déchirant la nuit urbaine, est la réponse d'un homme sans illusions sur l'espèce humaine mais qui ne peut s'empêcher de la trouver fascinante. Comme si avoir tout vu ne suffisait pas à assouvir sa passion de l'existence, le réalisme noir de ses images était aussi une façon de célébrer ce nouveau monde où il était devenu quelqu'un.

Avec Cindy Sherman, la boucle est bouclée ou plus exactement la transformation s'est complètement opérée. Il n'y a au fond même plus de sujet, plus que la photographe, puisque celle qui se donne comme une plasticienne contemporaine a, depuis ses débuts au milieu des années 70, fait de son corps son œuvre, répétant inlassablement le même protocole d'autoportrait transformiste. Le MoMA lui consacre une copieuse rétrospective où l'on peut découvrir les différentes séries, depuis les photogrammes («Film Stills») jusqu'aux immenses pseudo «society portraits» de la dernière 
décennie, tableaux de plus de trois mètres de haut où elle s'incarne en bourgeoises fortunées d'âge mur. On a beaucoup écrit sur Cindy Sherman à la fois pour donner une profondeur psychologique, voire philosophique à une œuvre qui n'en a peut-être pas autant qu'on veut le croire, et, plus rarement il est vrai, pour critiquer sa position dominante dans le marché de l'art. Le plus simple pour en juger est de parcourir cette rétrospective ou de feuilleter le livre qui en est tiré (et qui s'ajoute aux autres dithyrambes dont elle n'a cessé de faire l'objet). La sanction est alors claire: maniérisme sans grand attrait, facilités picturales et absence de teneur intellectuelle s'y révèlent avec évidence. Ce que le marché a monté en épingle (et en cotation...) ne résiste pas à l'accumulation que le MoMA a imaginé comme célébration et bien sûr comme blockbuster, tablant sur le "phénomène" Cindy Sherman qui continue d'emballer le public et de remplir ses caisses. Ce qui peut amuser (ou même plaire) à petite dose, perd tout intérêt une fois étalé à haute dose sur les murs d'un musée tel que le MoMa qui confirme ainsi son rôle d'arbitre du goût, pour le meilleur ou pour le pire, mais que l'on regrette de voir ainsi encenser une œuvre très largement surfaite.

Cette exposition peut simplement agacer. Vue à côté des deux autres, elle scandalise ou simplement inquiète. Les institutions de l'art contemporain semblent décidément bien malades pour promouvoir ainsi des travaux aussi fabriqués que ceux de Sherman (où l'on fait la grande découverte de la vacuité du présent...). Les photographes de la Photo League n'étaient peut-être que de doux rêveurs dans un monde qui n'était pas encore blasé. Mais comment, à quelques encablures des protestataires de Wall Street, à quelques rues de feu le World Trade Center, consacrer une nouvelle exposition à cette œuvre que des conservateurs complices essaient de faire passer aujourd'hui pour de l'art?

Alors, pour tous ceux pour qui la photographie et l'art ont encore un sens contemporain, il faut vite aller voir la Photo League, ne serait-ce qu'en lisant le très beau catalogue qui accompagne une des grandes expositions de la saison.

\section{Catalogues et références :}

KLEIN Mason et Catherine EVANS, The Radical Camera : New York's Photo League, 1936-1951, Textes de Maurice Berger, Michael Lesy, Anne Wilkes Tucker, New Haven, Conn., Yale University Press, 2011. 248 p.

WEEGEE, Naked City. New York, Da Capo, 2010.243 p.

RESPINI Eva, Cindy Sherman, New York, NY, Museum of Modern Art, 2012. 
INDEX

Thèmes : Trans'Arts

AUTEUR

JEAN KEMPF

Université Lumière Lyon 2 